\title{
Surface-enhanced Raman spectroscopy of blood serum based on gold nanoparticles for the diagnosis of the oral squamous cell carcinoma
}

Yingyun Tan ${ }^{1,2,3}$, Bing Yan ${ }^{4}$, Lili Xue ${ }^{5}, \mathrm{Yi} \mathrm{Li}^{6}$, Xianyang Luo ${ }^{4}$ and Ping Ji, $\mathrm{j}^{1,2,3^{*}}$

\begin{abstract}
Background: Oral squamous cell carcinoma (OSCC) is becoming more common across the globe. The prognosis of OSCC is largely dependent on the early detection. But the routine oral cavity examination may delay the diagnosis because the early oral malignant lesions may be clinically indistinguishable from benign or inflammatory diseases. In this study, the new diagnostic method is developed by using the surface enhanced Raman spectroscopy (SERS) to detect the serum samples from the cancer patients.
\end{abstract}

Method: The blood serum samples were collected from the OSCC patients, MEC patients and the volunteers without OSCC or MEC. Gold nanoparticles(NPs) were then mixed in the serum samples to obtain the high quality SERS spectra. There were totally 135 spectra of OSCC, 90 spectra of mucoepidermoid carcinoma (MEC) and 145 spectra of normal control group, which were captured by SERS successfully. Compared with the normal control group, the Raman spectral differences exhibited in the spectra of OSCC and MEC groups, which were assigned to the nucleic acids, proteins and lipids. Based on these spectral differences and features, the algorithms of principal component analysis(PCA) and linear discriminant analysis (LDA) were employed to analyze and classify the Raman spectra of different groups.

Results: Compared with the normal groups, the major increased peaks in the OSCC and MEC groups were assigned to the molecular structures of the nucleic acids and proteins. And these different major peaks between the OSCC and MEC groups were assigned to the special molecular structures of the carotenoids and lipids. The PCA-LDA results demonstrated that OSCC could be discriminated successfully from the normal control groups with a sensitivity of $80.7 \%$ and a specificity of $84.1 \%$. The process of the cross validation proved the results analyzed by PCA-LDA were reliable.

Conclusion: The gold NPs were appropriate substances to capture the high-quality SERS spectra of the OSCC, MEC and normal serum samples. The results of this study confirm that SERS combined PCA-LDA had a giant capability to detect and diagnosis OSCC through the serum sample successfully.

Keywords: Oral squamous cell carcinoma (OSCC), Surface-enhanced Raman spectroscopy (SERS), Principal component analysis (PCA), Linear discriminant analysis(LDA), Diagnosis

\footnotetext{
* Correspondence: Pingjicq@163.com

${ }^{1}$ Stomatological Hospital of Chongqing Medical University, Chongqing 400000, China

${ }^{2}$ Chongqing Key Laboratory of Oral Diseases and Biomedical Sciences, Chongqing 400000, China

Full list of author information is available at the end of the article
}

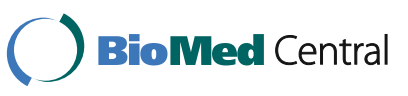

(c) The Author(s). 2017 Open Access This article is distributed under the terms of the Creative Commons Attribution 4.0 International License (http://creativecommons.org/licenses/by/4.0/), which permits unrestricted use, distribution, and reproduction in any medium, provided you give appropriate credit to the original author(s) and the source, provide a link to the Creative Commons license, and indicate if changes were made. The Creative Commons Public Domain Dedication waiver (http://creativecommons.org/publicdomain/zero/1.0/) applies to the data made available in this article, unless otherwise stated. 


\section{Background}

Oral squamous cell carcinoma (OSCC) is among the 10th most common cancer in the world, and the annual incidence of OSCC continues to increase specifically in Western and Asian countries [1, 2]. It is reported 3.29 per 100,000 as incidence rate and 1.49 per 100,000 as mortality rate in China [3]. The survival of OSCC is poor, with a 5-years survival rate of approximately $50 \%$ and has not a remarkable improve in the recent decades. The early detection by screening can be considered the best method to improve survival [4]. However the oral cavity is accessible to physical examination, the clinical visual examinations occasionally may delay the diagnosis because the early oral malignant lesions may be clinically indistinguishable from benign or inflammatory diseases [5]. The reliability of visual examination is also questionable and there are insufficient evidence to recommend a visual examination in screening for oral cancer in a lowrisk population $[5,6]$. The oral biopsy is considered the golden standard of OSCC diagnosis, but it is time consuming, labor intensive and invasive [7]. So a real-time, accurate and non-invasive diagnostic method is a pressing need for OSCC detection.

The surface-enhanced Raman spectroscopy (SERS) was first discovered by Fleichman and his colleages who founded the Raman intensities were enhanced as much as $10^{5}-10^{14}$ times by adsorbing molecules onto nanostructured metal surfaces [8-10]. SERS can overcome the drawbacks of regular Raman spectroscopy such as weak Raman intensities and strong autofluorescence background, and make the possibility of detecting trace or even single molecule. The SERS have been applied successfully on the detection of oncological diseases in different sites [11]. The key point of successful diagnosis by Raman spectroscopy is the dependence on the visual detection of morphologically or structurally aberrant lesions [2]. But the early oral carcinoma may be visually indistinguishable due to the subtle morphological alterations, and the Raman spectroscopic technique can not focus exactly on the lesions which could result in the false diagnosis. And some OSCC arising on the hidden sites in oral cavity such as the tongue base are not easy to be diagnosed at the early stage. So the spectral detection and analysis of biofluids using SERS is considered a novel, non-invasive and convenient method for the OSCC diagnosis. A systematic study based on the SERS of blood plasma and serum recommends the serum as the sample of choice due to the interference of anticoagulants in common plasma and various pathological metabolites in patients' serums [12]. In our previous study, SERS combined with support vector machine has successfully distinguished the patients with different parotid gland tumors from the normal subjects by detecting the blood serums [10]. So in this study, we developed the method of blood serum detection by using SRES based on gold nanoparticles to diagnose the OSCC.

\section{Methods \\ Subjects and protocol}

In this study, a total of 135 patients with OSCC were selected as the experimental group, who were not treated prior to this study and didn't have any other sysmetic diseases or drug abuse. In order to demonstrate the ability of discrimination of the method, 90 patinets with the mucoepidermoid carcinoma (MEC) were selected as the positive control group. And a total of 145 patients with old maxillofacial fracture and healthy volunteers were selected as the normal control group. More information on the subjects was shown in Table 1. At the beginning of the study, all subjects were informed detailedly and gave their written informed consents. The routine blood examinations of all subjects remained in the normal range. And this study was approved by the Ethics Committees of the First Affiliated Hospital of Xiamen University and followed the guidelines of the Helsinki Declaration.

\section{Preparation of the gold nanoparticles}

Gold nanoparticles(NPs) were prepared through the deoxidizing process reported in the our previous study [9]. A total of $0.7 \mathrm{ml}$ of $1 \% w / v$ trisodiumcitric acid was added rapidly in the beaker of rolling boiled $100 \mathrm{ml}$ $\mathrm{HAuCl}_{4}$. The mixed solution was heated to keep boiling and stirred continuously for half hours. The golden NPs were produced when the color of the solution changed from pale yellow to burgundy. The experimental conditions of different batches were controlled very carefully to guarantee the consistency of the shape and volume of the gold NPs. The gold NPs solution was stored at $4{ }^{\circ} \mathrm{C}$ for the SERS measurement.

\section{Preparation of blood serum samples}

A total of $5 \mathrm{ml}$ peripheral blood sample was obtained from the subject who had fasted overnight for $10 \mathrm{~h}$. The obtained blood sample was deposited at $4{ }^{\circ} \mathrm{C}$ for $4 \mathrm{~h}$

Table 1 Information on these subjects in this study

\begin{tabular}{llll}
\hline Information & Group & & Normal \\
\cline { 2 - 4 } & OSCC & MEC & \\
\hline Age(year) & & & $18-68$ \\
Age rang & $39-70$ & $28-79$ & 36 \\
Median age & 58 & 44 & \\
Gender & & & 93 \\
Male & 75 & 50 & 52 \\
Female & 60 & 40 & 145 \\
Total & 135 & 90 & \\
\hline
\end{tabular}


without any anticoagulant. Then the blood sample was centrifuged at $3400 \mathrm{rpm}$ for $10 \mathrm{mins}$ in order to remove the cells, fibrinogen and platelet in the blood. A single $1 \mathrm{ml}$ supernatant serum sample was collected from the centrifuged blood and stored at $-20{ }^{\circ} \mathrm{C}$ the Raman detection.

\section{Scanning electron microscopy (SEM) and the ultraviolet- visible spectroscopy analysis}

The morphologies of the gold NPs were detected by using a scanning electron microscope (HITACHI S4800, Hitachi Ltd., Tokyo, Japan) with a voltage of $30 \mathrm{kV}$. The absorption of the gold NPs and the mixture of the serum and gold NPs were monitored by the ultraviolet-visible spectroscope (Cary5000, VARIAN Ltd., USA).

\section{SERS measurement}

A total $4 \mathrm{ml}$ of gold NPs solution prepared in the above processing was added into a tube and centrifuged at $6000 \mathrm{rpm}$ for $10 \mathrm{mins}$. Then the supernatant was discarded from the gold NPs and a $0.4 \mathrm{ml}$ serum sample was added for the SERS measurement. The mixed solution was vibrated by ultrasonic oscillator in order to make the NPs distribute more homogeneously. The solution was incubated at $4{ }^{\circ} \mathrm{C}$ for $2 \mathrm{hs}$ before the SERS measurement. The SERS measurement was carried out by using a Renishaw inVia Raman microscope (Renishaw Ltd., UK) with a $633 \mathrm{~nm}$ laser. The excitation laser with a power of about $0.4 \mathrm{~W}$ was focused on the serum samples through a $\times 50$ objective lens $(\mathrm{NA}=0.75)$. The spectra were recorded in the $200-1800 \mathrm{~cm}^{-1}$ Raman shift range with a $2 \mathrm{~cm}^{-1}$ spectral resolution. Every spectrum was integrated for $10 \mathrm{~s}$ and averaged over 2 accumulations.

\section{Data pre-processing}

Before the data statistical analysis, the raw Raman spectral data were preprocessed by WiRE 2.0 software (Renishaw Ltd., UK) to remove the noisy interferences and oversaturated spectra. The autofluorescence backgrounds were removed by the 4th degree polynomial function and the SERE spectra were smoothed by the Savitzky-Golay smoothing through the LABSPEC 2.0 software (HORIBA Scientific, France). Then the baseline correction and normalization were carried out before the further analysis and comparison of the different spectra.

The mean spectra of different groups were obtained by calculating and analyzing the pre-processed data through the OringinPro 8.0 software (OringinLab, USA). The spectral differences between the groups were obtained by subtracting the mean spectra of different groups. The differences of peaks shown in the subtracted spectra were assigned to the molecular structures and biochemical component based on the results reported in the previous literatures.

\section{Multivariate analysis}

Principal component analysis(PCA) was employed to reduce the dimensions and determine the key variables. In this study, the retaining principal components which accounted for $90 \%$ of the variance in the spectral data was applied as statistical variables and input into Linear discriminant analysis(LDA) for the serum classification. The prediction performance of the diagnostic model rendered by LDA was estimated and evaluated by the leave-one-out-cross-validation method. This study discriminated the OSCC group from the normal group firstly, and then discriminated the OSCC group from the MEC group and the normal group.

\section{Results}

The SEM image of the pure gold NPs was shown in the Fig. 1a. The spherical NPs with a mean diameter of $55 \mathrm{~nm}$ were prepared in this method and had a maximal absorption at $530 \mathrm{~nm}$. In the UV-visible absorption spectra shown in Fig. 1b, the pure NPs solution absorption band appeared in the around $530 \mathrm{~nm}$ wavelength region, and the band of the mixture of serum and NPs also remained in the same region but the intensity reduced due to the combination of gold NPs and biochemical substances in the serum. Compared with the regular Raman spectrum, the intensities of the SERS spectrum were enhanced enormously due to the gold NPs added in the serum (Fig. 1c).

A total of 370 SERS spectra were recorded successfully in the Raman shift region from $200 \mathrm{~cm}^{-1}$ to $1800 \mathrm{~cm}^{-1}$. Among these spectra, 135 spectra were obtained from the clinically confirmed OSCC subjects, 90 spectra were obtained from the clinically confirmed MEC subjects and 145 spectra were obtained from the normal control subjects. The clinical data of the subjects participating in this study was shown in the Table 1. The mean spectra of different groups before the spectral normalization were presented in the Fig. 2. And after the spectral normalization, the normalized mean spectra were used to compare the spectral differences of the OSCC, MEC and normal groups (Fig. 3). Compared with the mean spectrum of the normal control group, the OSCC group showed the increase in the peaks at $294,446,548,726$, $745,1136,1263,1371,1445$ and $1491 \mathrm{~cm}^{-1}$ but the decrease in the peaks at 1542 and $1602 \mathrm{~cm}^{-1}$, which were shown in the subtracted spectrum (Fig. 4a). Compared with the mean spectrum of the normal control group, the MEC group showed the increase in the peaks at 476, 548, 726, 745, 933, 1328, 1371 and $1445 \mathrm{~cm}^{-1}$ but the decrease in the peaks at 294,1263, 1541 and $1607 \mathrm{~cm}^{-1}$ (Fig. 4b). The spectral differences were also presented in 

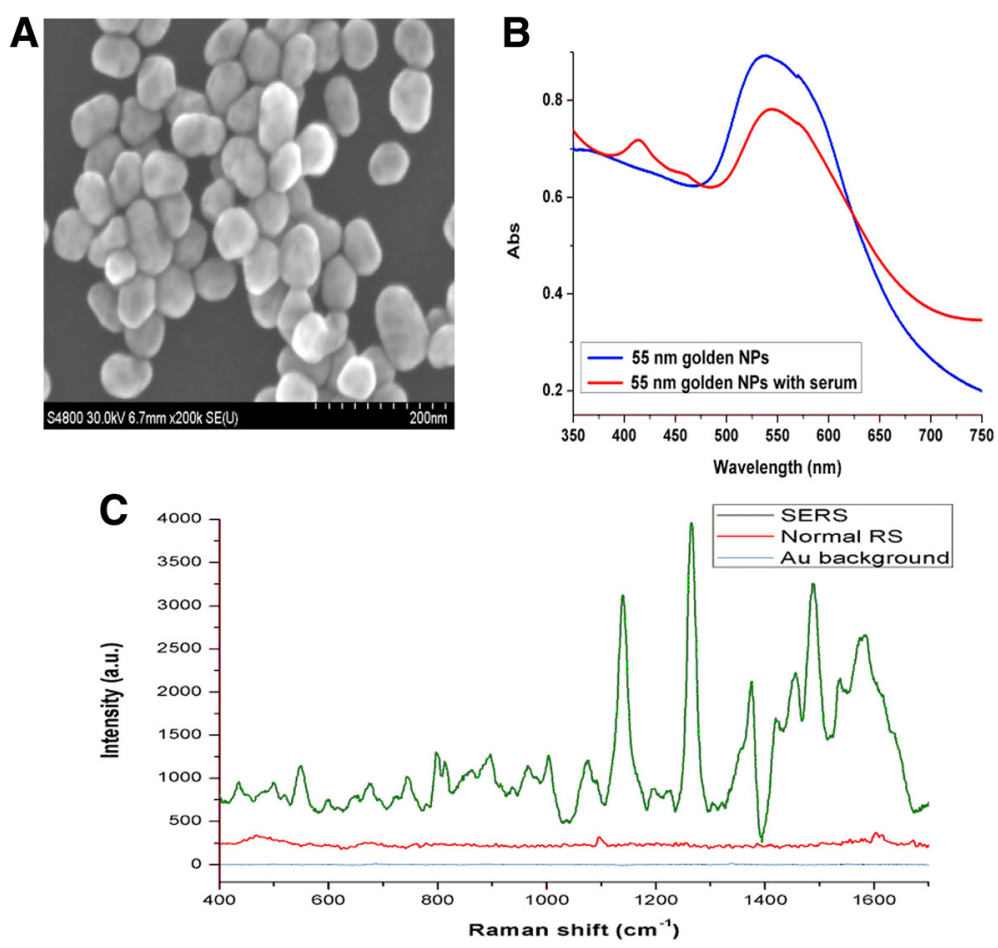

Fig. 1 The SEM image, absorption curve and Raman spectrum of the gold NPs. a The SEM image of the gold NPs. b The UV/visible absorption of the gold NPs and the gold NPs with serum. c SERS spectrum of serum, routine Raman spectrum of serum and background Raman spectrum of the gold NPs

the subtracted spectrum between the OSCC group and MEC group. The subtracted spectrum showed the increase in the peaks at $294,1139,1263$ and $1491 \mathrm{~cm}^{-1}$ but the decrease in the peaks at $1602 \mathrm{~cm}^{-1}$ in the OSCC group compared with the MEC group (Fig. 4c).

All these peaks of different intensities shown in the subtracted spectrum can be assigned to the various biochemical substances and molecular structures according to the reported literatures and previous studies $[9,13-18]$ (Table 2). Compared with the normal groups, the major increased peaks in the OSCC and MEC groups were assigned to the molecular structures of the nucleic acids and proteins. And these different major peaks between the OSCC and MEC groups were
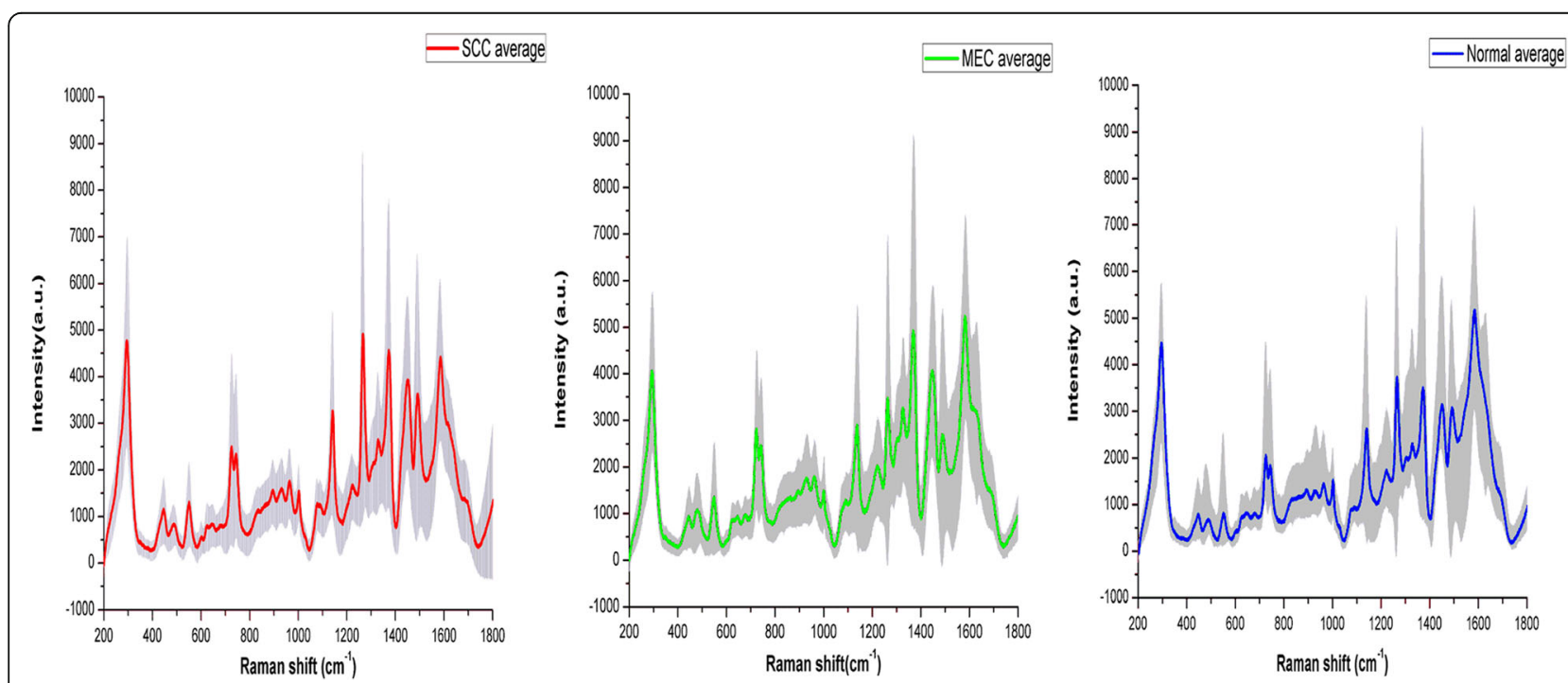

Fig. 2 The average Raman spectra of OSCC, MEC and normal serum samples. The gray areas manifest the standard deviations 


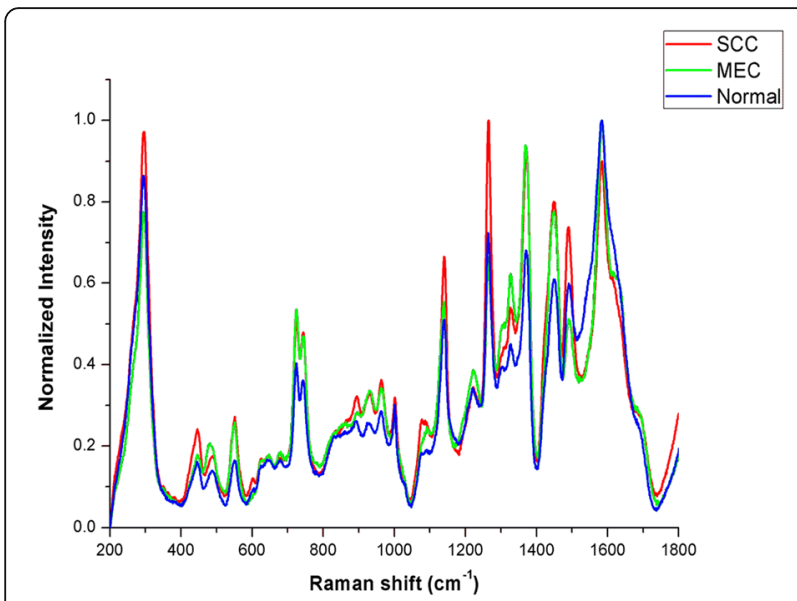

Fig. 3 The normalized mean Raman spectra of OSCC, MEC and normal serum samples

assigned to the special molecular structures of the carotenoids and lipids. The diagnostic classification of the different spectra can be carried out based on these peaks reported above.

In the first analytical step to discriminate the OSCC spectra from the normal ones, the OSCC spectra were selected as the positive group and the normal ones were selected as the negative group. The process of PCA extracted 55 principle components(PCs) from the raw spectral data, which captured about $95 \%$ of the cumulative variance of the raw data and were input as variables for the LDA process. As the result of the LDA, 109 of 135 OSCC spectra and 122 of 145 normal spectra were classified into the accurate group successfully (Table 3). The sensitivity and specificity were 80.7 and $84.1 \%$ respectively, and the total accuracy of this diagnostic classification was $82.5 \%$. The histogram of discrimination scores demonstrated a clear classification of the two groups (Fig. 5). In order to test the results of the classification, the 'leave-one-out' method was employed in the cross validation process. And the result of the cross validation shown that $107 / 135$ of OSCC spectra and 120/145 of normal spectra were diagnosed correctly (Table 4). The sensitivity and specificity of the diagnosis were 79.3 and $82.8 \%$, and the total accuracy was $81.1 \%$.

In order to demonstrate the potential more effectively to diagnose the OSCC by SERS, the MEC spectra were selected as the positive control group in the diagnostic classification. The PCA process also extracted 51 PCs from the raw data, which accounted for about $93 \%$ of the total variance. Then the PCs were input in the LDA process to classify the three different groups. The result shown that 101/ 135 of OSCC spectra, 72/90 of MEC spectra and 129/145 of normal spectra were classified into the correct groups successfully (Table 5). The sensitivity and specificity of the diagnosis of OSCC were 74.8 and $89.0 \%$, and the total
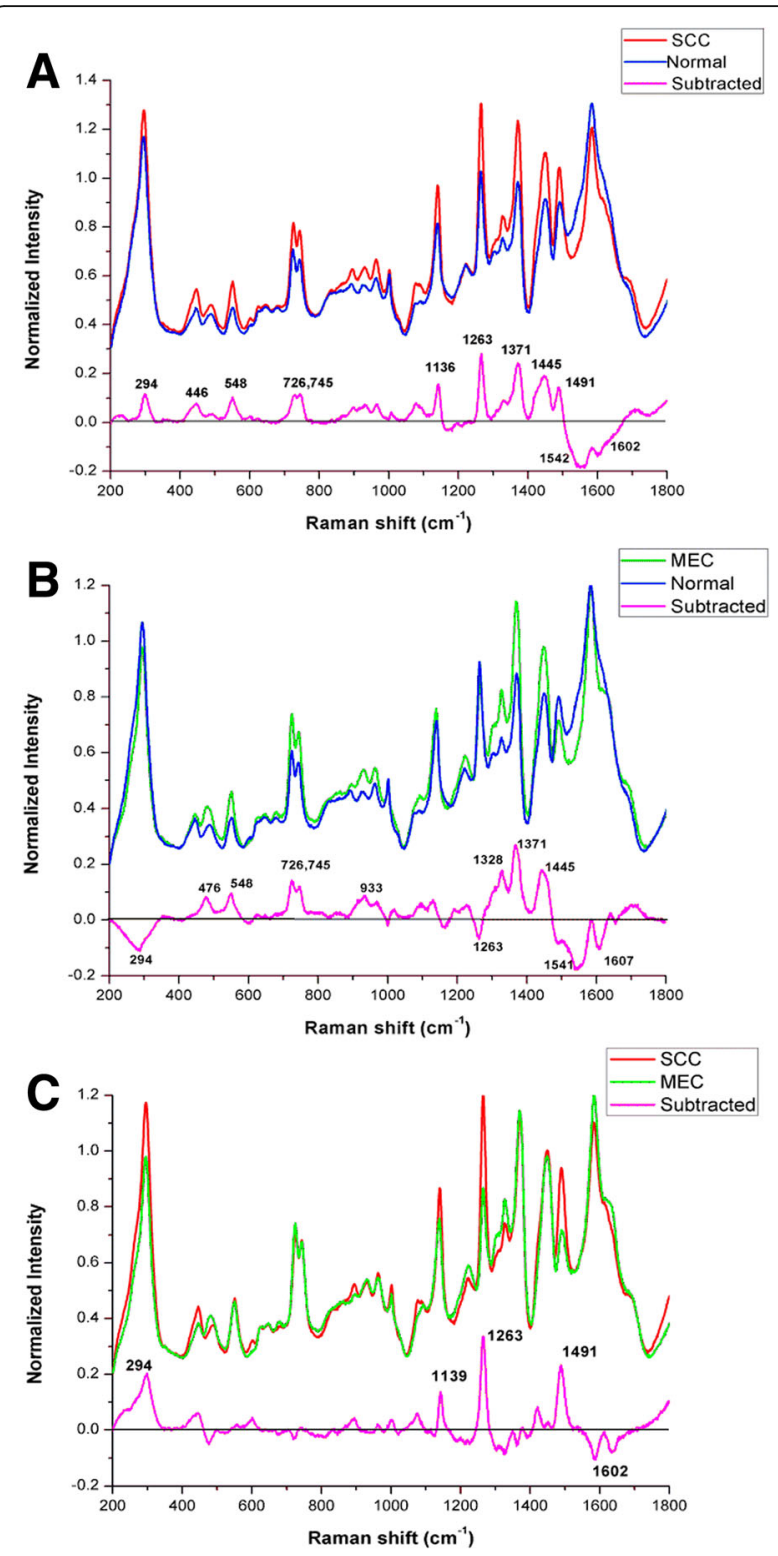

Fig. 4 The subtracted spectra of the OSCC, MEC and normal serum samples

accuracy was $81.6 \%$. The scatter plot diagram showed the separation of the three groups (Fig. 6). Then the 'leave-oneout' method was employed in the cross validation process to test the results of the classification. In the results of the cross validation, $82 / 135$ of OSCC spectra were classified correctly, 64/90 of MEC spectra and 109/145 of normal spectra were also diagnosed correctly. The sensitivity and specificity of the diagnosis of OSCC were 60.7 and 75.2\%, and the total accuracy was $68.9 \%$ (Table 6).

\section{Discussion}

The American Academy of Oral Medicine recognized that the patients with oral cancer detected in the early 
Table 2 Raman shifts of peaks and the characteristic assignments

\begin{tabular}{ll}
\hline Raman shift $\left(\mathrm{cm}^{-1}\right)$ & Peak assignment \\
\hline 294 & Au-S band \\
$446-476$ & Cholesterol \\
548 & S-S disulfide stretching in Proteins \\
726 & Hypoxanthine \\
745 & Thymine in DNA \\
933 & $\mathrm{C}-\mathrm{C}$ stretching mode, C-C ahelix in proteins \\
$1136-1139$ & $\mathrm{C}-\mathrm{N}$ stretch in D-mannons \\
1263 & $\mathrm{CH}$ bending in lipids \\
1328 & $\mathrm{CH}$ vibration in DNA/RNA, $\mathrm{CH}_{2}$ twisting in lipids \\
1371 & $\mathrm{Guanine}$ in DNA, Tryptophan in proteins \\
1445 & $\mathrm{CH}, \mathrm{CH}_{3}$ bending in proteins and lipids \\
1491 & $\mathrm{CH}$ bending \\
$1541-1542$ & $\mathrm{C}-\mathrm{N}$ stretching, Amide II \\
$1602-1607$ & $\mathrm{C}=\mathrm{C}$ band in Phenylalanine or Tyrosine \\
\hline
\end{tabular}

stages required less aggressive treatment and experienced fewer complications than the ones with advanced stage cancers, and the patients with early stage oral cancer would have improved survival [19]. And the evidence existed in a systematic review that the development and use of biomarkers was becoming increasingly common in the early detection of oral cancer [20]. The metabolites in the biofluids of the patients with cancers are different from the normal subjects, due to the amino acid metabolism, cell apoptosis and tumor necrosis, which can be used for the cancer detection as the biomarkers $[10,12]$. The serum or plasma have been selected as the samples for the cancer detection and diagnosis by Raman spectroscopy successfully because the serum or plasma is easily collected and mostly contains some vital biomarkers generated by cancers $[10,15,21,22]$. Rekha et al. [21] reported that the plasma Raman spectra of the oral premalignant and malignant conditions were different from the normal condition, and could be utilized to diagnose the oral premalignant and malignant conditions with the sensitivity and specificity of above $80.0 \%$. Serum Raman spectroscopy had the potential not only in the diagnosis of oral cancers, but also in the prediction of treatment responses [22]. Accordingly, the serum was the sample of the choice in this study. The SERS has been employed to detect and diagnose the cancers

Table 3 The results of the classification of OSCC and normal group

\begin{tabular}{|c|c|c|c|c|}
\hline \multirow[t]{2}{*}{ Class } & \multicolumn{3}{|c|}{ Predicted group } & \multirow[t]{2}{*}{ Total } \\
\hline & & oscC & Normal & \\
\hline \multirow[t]{2}{*}{ Count(\%) } & oscC & 109(80.7\%) & $26(19.3 \%)$ & 135(100\%) \\
\hline & Normal & 23(15.9\%) & $122(84.1 \%)$ & $145(100 \%)$ \\
\hline
\end{tabular}

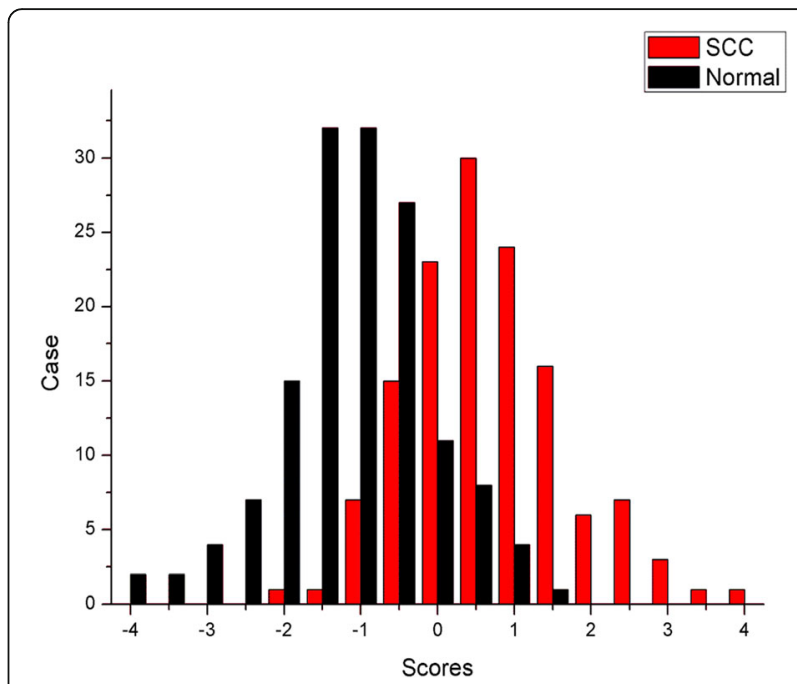

Fig. 5 The histogram of discrimination scores of OSCC and normal group

with a high accuracy in different sites. High-quality SERS of serum was used to identify the colorectal cancer with the diagnostic accuracy of $100 \%$ [15]. The SERS in this study successfully made the serum constituents adsorb on the nanoparticles and captured the biomolecular spectra fingerprints of the biomarkers.

In this study, the spectral differences of the peaks assigned to various biomolecular fingerprints were captured in the SERS. Compared with the normal control group, the spectra of OSCC and MEC group exhibited the increasing intensities of the peaks at the $446 \sim 476$, $548,726,745,1371$ and $1445 \mathrm{~cm}^{-1}$. These peaks were assigned to the nucleic acids and proteins which could be caused by the active metabolism of nucleic acids in the patients with cancers $[10,22]$. These findings were similar to the results reported in the previous studies. Rekha et al. [21] and Sahu et al. [22] all found that the increasing intensities of peaks at 1339 and $1445 \mathrm{~cm}^{-1}$ were related to the increase of the nucleic acids and proteins in the plasma and serum of the oral cancer groups. But in their reported studies, the ranges of Raman shifts were limited from the $700 \mathrm{~cm}^{-1}$ or $800 \mathrm{~cm}^{-1}$ to $180 \mathrm{~cm}$ ${ }^{-1}$, and the only routine Raman spectra were obtained, so there were some differences between their results and ours. Feng et al. [23] reported that the SERS band at

Table 4 The results of the cross validation of OSCC and normal group

\begin{tabular}{|c|c|c|c|c|}
\hline \multirow[t]{2}{*}{ Class } & \multicolumn{3}{|c|}{ Predicted group } & \multirow[t]{2}{*}{ Total } \\
\hline & & oscC & Normal & \\
\hline \multirow[t]{2}{*}{ Count(\%) } & OsCC & 107(79.3\%) & 28(20.7\%) & 135(100\%) \\
\hline & Normal & $25(17.2 \%)$ & 120(82.8\%) & $145(100 \%)$ \\
\hline
\end{tabular}


Table $\mathbf{5}$ The results of the classification of OSCC, MEC and normal group

\begin{tabular}{llllll}
\hline Class & & \multicolumn{2}{l}{ Predicted group } & \multirow{2}{*}{ Total } \\
\cline { 3 - 4 } & & OSCC & MEC & Normal & \\
\hline Count(\%) & OSCC & $101(74.8 \%)$ & $19(14.1 \%)$ & $15(11.1 \%)$ & $135(100 \%)$ \\
& MEC & $3(3.3 \%)$ & $72(80.0 \%)$ & $15(16.7 \%)$ & $90(100 \%)$ \\
& Normal & $8(5.5 \%)$ & $8(5.5 \%)$ & $129(89.0 \%)$ & $145(100 \%)$ \\
\hline
\end{tabular}

$725 \mathrm{~cm}^{-1}$ were greater in nasopharyngeal cancer plasma than the ones in the normal plasma, and the band was assigned to the nucleic acids. The increasing proteins in the serum of OSCC and MEC could result from the redistribution or translocation of free amino acids in the blood of the patients with cancers [24]. Compared with the MEC group, there were the increasing intensities of peaks at $1139,1263,1491 \mathrm{~cm}^{-1}$ and the decreasing intensity of peak at $1602 \mathrm{~cm}^{-1}$ in the SERS of OSCC group. These differences demonstrated the higher D-mannos and lipids level in the OSCC group. The previous study reported that there was an alteration in plasma lipid constituents in cancer patients because the cancer cells would utilize lipids for new membrane biogenesis [25]. So all above spectral differences captured in the SERS could be employed as the diagnostic indicators and references to detect the OSCC serum samples.

In order to develop and establish the classification and diagnostic models, numerous algorithms were employed to analyze Raman spectral data in the reported studies [21, 26-28]. Among these algorithms, PCA was a statistical technique for reducing the dimensions and simplifying complex data sets with the minimum reconstruction error [29]. However, PCA was not suitable for classification problems because it could not use any class information in computing the features extracted from the original data [30]. So Belhumeur et al. [31] combined PCA with LDA to make a popular

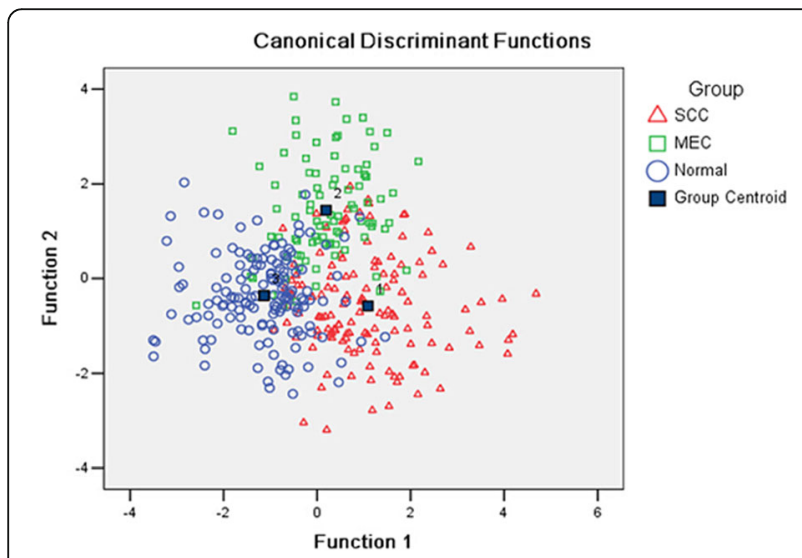

Fig. 6 The scatter plot of discrimination scores of OSCC, MEC and normal group
Table 6 The results of the cross validation of OSCC, MEC and normal group

\begin{tabular}{llllll}
\hline Class & & \multicolumn{2}{l}{ Predicted group } & \multirow{2}{*}{ Total } \\
\cline { 3 - 5 } & & OSCC & MEC & Normal & \\
\hline Count(\%) & OSCC & $82(60.7 \%)$ & $27(20.0 \%)$ & $26(19.3 \%)$ & $135(100 \%)$ \\
& MEC & $9(10.0 \%)$ & $64(71.1 \%)$ & $17(18.9 \%)$ & $90(100 \%)$ \\
& Normal & $19(13.1 \%)$ & $17(11.7 \%)$ & $109(75.2 \%)$ & $145(100 \%)$ \\
\hline
\end{tabular}

method for dimension reduction and classification of data sets. LDA was a well-known method which found a linear transformation such that feature clusters were most seperable after the transformation [29]. The PCA combined with LDA were employed to classify and diagnose the different disease successfully based on the Raman spectral features $[18,23,26,32]$. Rekha et al. [21] used PCA-LDA to yield a diagnostic sensitivity of $91.2 \%$ and a specificity of $96.7 \%$ in the classification of normal from oral malignant group. In this study, the PCA-LDA could also successfully classify and diagnose the spectra of OSCC and normal group with a sensitivity of $80.7 \%$ and a specificity of $84.1 \%$. The results of the cross validation demonstrated the utilization of the PCA-LDA algorithm in the analysis of Raman spectral data was reliable. Then PCA-LDA had an excellent performance in the analysis and classification of the spectra of the OSCC, MEC and normal groups with a total accuracy of $68.9 \%$, which showed a giant potential to diagnose OSCC based on the SERS.

\section{Conclusion}

The gold NPs were appropriate substances to capture the high-quality surface enhanced Raman spectra of the OSCC, MEC and normal serum samples. In these Raman spectra, there were differences assigned to some specific molecular structures and components, which reflect the different levels of nucleic acids, proteins and lipids in the cancers and normal serums. These differences might result from the metabolic alterations, cell proliferation and apoptosis in the cancer serum. Based on these spectral differences and features, PCA-LDA could classify and diagnose the OSCC, MEC and normal groups successfully. In a conclusion, SERS combined PCA-LDA had a giant capability to detect and diagnosis OSCC through the serum sample successfully.

\section{Abbreviations}

LDA: linear discriminant analysis; MEC: mucoepidermoid carcinoma; NPs: Gold nanoparticles; OSCC: oral squamous cell carcinoma; PCA: principal component analysis; SEM: scanning electron microscopy.; SERS: surface enhanced Raman spectroscopy

\section{Acknowledgements}

The authors like to thank the Prof. Bin Ren and Dr. Chen Zong for their technical support. 


\section{Funding}

This work is supported by the National Science Foundation of China (Grant No. 81172578), the Project of Medical innovation of Fujian Province (Grant No. 2015-CXB-35) and the Project of Science and Technology of Xiamen City (Grant No. 3502Z20134007). The funders had no role in the design of the study and collection, analysis, interpretation of data and preparation of the manuscript.

\section{Availability of data and materials}

All data reported in this study are included in this published article. Further details can be available from the corresponding author on request.

\section{Authors' contributions}

YT, BY and PJ conceived and designed this study. YT and BY contributed in the preparation of NPs and SERS measurement. $L X$ and $Y L$ carried out the preparation of serum samples. YT, BY and $\mathrm{XL}$ carried out the data analysis. YT, BY and PJ prepared the manuscript. All authors read and approved the final manuscript.

\section{Competing interests}

All authors agree to the submission of this research for publication and declare that there are no conflict of interests.

\section{Consent for publication}

Not applicable.

\section{Ethics approval and consent to participate}

This study was approved by the Ethics Committees of the First Affiliated Hospital of Xiamen University (Reference No. KY2013-01 ).

\section{Publisher's Note}

Springer Nature remains neutral with regard to jurisdictional claims in published maps and institutional affiliations.

\begin{abstract}
Author details
${ }^{1}$ Stomatological Hospital of Chongqing Medical University, Chongqing 400000, China. ${ }^{2}$ Chongqing Key Laboratory of Oral Diseases and Biomedical Sciences, Chongqing 400000, China. ${ }^{3}$ Chongqing Municipal Key Laboratory of Oral Biomedical Engineering of Higher Education, Chongqing 400000, China. ${ }^{4}$ Department of Otolarygology Head and Neck Surgery, the First Affiliated Hospital of Xiamen University, Xiamen 361000, China. ${ }^{5}$ Department of Stomatology, the First Affiliated Hospital of Xiamen University, Xiamen 361000, China. ${ }^{6}$ Department of Head and Neck Oncology, the West China Hospital of Stomatology, Sichuan University, Chengdu 610000, China.
\end{abstract}

Received: 14 February 2017 Accepted: 30 March 2017

Published online: 07 April 2017

\section{Reference}

1. Lee C-C, Hsu-Chueh H, Yu-Chieh S, Po-Chun C, Yu C-H, Yang C-C Comparison of different comorbidity measures for oral cancer patients with surgical intervention: a longitudinal study from a single cancer center. Auris Nasus Larynx. 2016;43:322-9.

2. Connolly JM, Davies K, Kazakeviciute A, Wheatley AM, Dockery P, Keogh I, Olivo M. Non-invasive and label free detection of oral squamous cell carcinoma using saliva surface-enhanced Raman spectroscopy and multivariate analysis. Nanomed: Nanotechnol Biol Med. 2016;12:1593-601.

3. Jin-Ye F, Gao J, Zhi-Yuan Z, Zheng J-W, Luo J-F, Lai-Ping Z, Yong-Bing X. Tea consumption and the risk of oral cancer incidence: a case-control study from China. Oral Oncol. 2013;49:918-22.

4. Radoi L, Luce D. A review of risk factors for oral cavity cancer: the importance of a standardized case definition. Community Dent Oral Epidemiol. 2013;41:97-109.

5. Rashid A, Warnakulasuriya S. The use of light-based (optical) detection system as adjuncts in the detection of oral cancer and oral potentially malignant disorders: a systematic review. J Oral Pathol Med. 2015;44:307-28.

6. Brockleburst P, Kujan O, Glenny AM, et al. Screening programmes for the early detection and prevention of oral cancer. Cochrane Database Syst Rev. 2010;10:CD004150.

7. Kumar S, Kumar S, Tiwari S, Augustine S, Srivastava S, Yadav BK, Malhotra $\mathrm{BD}$. Highly sensitive protein functionalized nanostructured hafnium oxide based biosensing platform for non-invasive oral cancer detection. Sensors Actuators B Chem. 2016;235:1-10.

8. Fleischman M, Hendra PJ, McQuillan AJ. Raman spectra of pyridine absorbed at a silver electrode. Chem Phys Lett. 1974;26:163-6.

9. Feng S, Chen R, Lin J, Pan J, Wu Y, Li Y, Chen J, Zeng H. Gastric cancer detection based on blood plasma surface-enhanced Raman spectroscopy excited by polarized light. Biosens Bioelectron. 2011;26:3167-74.

10. Yan B, Li B, Wen Z, Luo X, Xue L, Li L. Label-free blood serum detection by using surface-enhanced Raman spectroscopy and support vector machine for the preoperative diagnosis of parotid gland tumors. BMC Cancer. 2015; 15:650-8.

11. Kong K, Kendall C, Stone N, Notingher I. Raman spectroscopy for medicine diagnostics-from in-vitro biofluid assays to in-vivo cancer detection. Adv Drug Deliv Rev. 2015;89:121-34.

12. Bonifacio A, Marta SD, Spizzo R, Cervo S, Steffan A, Colombatti A, Sergo V. Surface-enhanced Raman spectroscopy of blood plasma and serum using Ag and au nanoparticles: a systematic study. Anal Bioanal Chem. 2014;406: 2355-65.

13. Agoston R, Izake EL, Sivanesan A, Lott WB, Sillence M, Steel R. Rapid isolation and detection of erythropoietin in blood plasma by magnetic core gold nanoparticles and portable Raman spectroscopy. Nanomed: Nanotechnol Biol Med. 2016;12:633-41.

14. Chen F, Flaherty BR, Cohen CE, Peterson DS, Zhao Y. Direct detection of malaria infected red blood cells by surface enhanced Raman spectroscopy. Nanomed: Nanotechnol Biol Med. 2016;12:1445-51.

15. Wang J, Lin D, Lin J, Yu Y, Huang Z, Chen Y, Lin J, Feng S, Li B, Liu N, Chen R. Label-free detection of serum proteins using surface-enhanced Raman spectroscopy for colorectal cancer screening. J Biomed Opt. 2014;19:087003.

16. Li X, Yang T, Li S, Wang D, Song Y, Yu K. Different classification algorithms and serum surface enhanced Raman spectroscopy for noninvasive discrimination of gastric diseases. J Raman Spectrosc. 2016;47:917-25.

17. Lin J, Wang J, Xu C, Zeng Y, Chen Y, Li L, Huang Z, Li B, Chen R. Differentiation of digestive system cancers by using serum protein-based surface-enhanced Raman spectroscopy. J Raman Spectrosc, 2016, DOI 10. 1002/jrs.4982.

18. Label-free blood plasma test based on surface-enhanced Raman scattering for tumor stages detection in nasopharyngeal cancer. Sci Rep, 2014; 4:4751.

19. Kerr RA. Oral cancer examination and screening. AAOM Clin Pract Statement. 2016. DOl:10.1016/j.00oo. 2016. 04. 009.

20. Brocklehurst P, Kujan O, O'Malley LA, Ogden G, Shepherd S, Glenny AM. Screening programmes for the early detection and prevention of oral cancer. Cochrane Database Syst Rev. 2013; Issue 11, Art.No.: CD004150. DOI: 10.1002/14651858.CD004150.pub4.

21. Rekha P, Aruna P, Bharaniaharan G, Koteeswaren D, Baludavid M, Ganesan S. Near infrared Raman spectroscopic characterization of blood plasma of normal, oral premalignant and malignant conditions-a pilot study. J Raman Spectrosc. 2015;46:735-43.

22. Sahu A, Nandakumar N, Sawant S, Krishna CM. Recurrence prediction in oral caners: a serum Raman spectroscopy study. Analyst. 2015;140:2294-301.

23. Feng S, Chen R, Lin J, Pan J, Chen G, Li Y, Cheng M, Huang Z, Chen J, Zeng $H$. Nasopharyngeal cancer detection based on blood plasma surfaceenhanced Raman spectroscopy and multivariate analysis. Biosens Bioelectron. 2010;25:2414-9.

24. Lai HS, LEE JC, Lee PH, Wang ST, Chen WJ. Plasma free amino acid profile in cancer patients. Semin Cancer Biol. 2005;15:267-76.

25. Patel PS, Shah MH, Jha FP, Raval GN, Rawal RM, Patel MM, Patel JB, Patel DD. Alterations in palsma lipid profile patterns in head and neck cancer and oral precancerous conditions. Indian J Cancer. 2004;41:25-31.

26. Xue L, Sun P, Ou D, Chen P, Chen M, Yan B. Diagnosis of pathological minor salivary glands in primary Sjogren's syndrome by using Raman spectroscopy. Lasers Med Sci. 2014;29:723-8.

27. Yan B, Li Y, Yang G, Wen Z, Li M, Li L. Discrimination of parotid neoplasms from the normal parotid gland by use of Raman spectroscopy and support vector machine. Oral Oncol. 2011:47:430-5.

28. Yan B, Wen Z, Li Y, Li L, Xue L. An intraoperative diagnosis of parotid gland tumors using Raman spectroscopy and support vector machine. Laser Phys. 2014;24:115601.

29. Zhou C, Lan W, Zhang Q, Wei X. Face recognition based on PCA image reconstruction and LDA. Optik. 2013;124:5599-603.

30. Lu GF, Zou J, Wang Y. Incremental complete LDA for face recognition. Pattern Recogn. 2012;45:2510-21. 
31. Belhumeur PN, Hespanha JP, Kriegman DJ. Eigenfaces vs. Fisher faces: recognition using class specific linear projection. IEEE Trans Pattern Anal Mach Intell. 1997;19:711-20.

32. Mistro G, Cervo S, Mansutti E, Spizzo R, Colombatti A, Belmonte P,

Zucconelli R, Steffan A, Sergo V, Bonifacio A. Surface-enhanced Raman spectroscopy of urine for prostate cancer detection: a preliminary study. Anal Bioanal Chem. 2015;407:3271-5.

Submit your next manuscript to BioMed Central and we will help you at every step:

- We accept pre-submission inquiries

- Our selector tool helps you to find the most relevant journal

- We provide round the clock customer support

- Convenient online submission

- Thorough peer review

- Inclusion in PubMed and all major indexing services

- Maximum visibility for your research

Submit your manuscript at www.biomedcentral.com/submit 